\title{
Diagnostic Approach to Suspected Perihilar Malignancy
}

\author{
Evgeny Solonitsyn ${ }^{a} \quad$ Alexander Dechêne ${ }^{b}$ \\ ${ }^{a}$ Almasov National Medical Research Centre, Saint-Petersburg, Russia; ${ }^{b}$ Department of Gastroenterology, \\ Hepatology and Endocrinology, University Hospital, Paracelsus Medical University Nuremberg, Nuremberg, \\ Germany
}

\author{
Keywords \\ Cholangiocarcinoma - Endoscopic retrograde \\ cholangiopancraticography · Cholangioscopy · \\ Endosonography · Biopsy
}

ically harvested) specimens is pivotal to differential diagnosis. Several new techniques to increase diagnostic accuracy are under investigation (biomarkers and genetic testing among others).

(c) 2021 S. Karger AG, Basel

\begin{abstract}
Background: Cholangiocarcinomas are relatively rare tumors most frequently arising from the epithelium of the hilar bile ducts. The diagnosis is often made in advanced (symptomatic) stages, which accounts for the infavorable prognosis with a 5 -year survival of less than $10 \%$. Identification of perihilar cholangiocarcinoma ( $p C C A$ ) is often challenging because there is no single method offering sufficient diagnostic accuracy. Summary: Most tumors arise in patients without specific risk factors. Clinical symptoms of pCCA are nonspecific and reliable biomarkers are lacking, with carbohydrate antigen 19-9 being the most commonly used tumor marker (but with a low accuracy). Cross-sectional imaging (CT and MRI) is used to identify and map hilar strictures and determine resectability by showing vascular involvement. Endoscopic ultrasound offers additional information on locoregional tumor spread and lymph node involvement. Endoscopic retrograde cholangiography in combination with cholangioscopy gives direct access to and imaging of hilar strictures but it does not always distinguish between pCCA and benign hilar strictures. Tissue acquisition for histological diagnosis is challenging, with frequent sampling errors regardless of the method of biopsy procurement because of the cellular paucity of tumor tissue. Key Messages: In suspected perihilar malignancy, a mosaic of clinical data has to be taken into account. Histological evaluation of (endoscop-
\end{abstract}

\section{Introduction}

Cholangiocarcinomas (CCA) are mostly adenocarcinomas arising from the epithelia of intra- or extrahepatic bile ducts, and they account for approximately $3 \%$ of all gastrointestinal malignancies in the Western world [1]. In about $50 \%$ of all cases, the tumor is located in the hepatic hilum (also called a Klatskin tumor). As benign lesions can mimic perihilar CCA (pCCA), the diagnosis of malignant disease is not always straightforward. Because (early) curative resection is the only curative treatment option for CCA to date, a timely differential diagnosis and accurate preoperative staging are the main aims in perihilar disease.

In this review, we provide an overview of the current diagnostic principles and options.

\section{Clinical Signs of Perihilar Malignancy}

The risk factors that predispose to CCA include intrabiliary parasitic disease, congenital biliary anomalies such as choledochal cysts, chronic and recurrent choledochoor cholangiolithiasis, recurrent biliary tract infections, and - most notably - primary sclerosing cholangitis [2]. 
Although there are no widely accepted concepts of screening in populations at high risk of developing CCA, a combination of repeat tumor marker evaluations and noninvasive imaging is used by many physicians [3].

In most patients with pCCA, jaundice is the first clinical sign of biliary congestion resulting from the obstruction of bile flow at the hilar level [4]. Other early, but entirely nonspecific, symptoms are weight loss, fatigue, abdominal discomfort, and pruritus. In virtually all patients, a rise in cholestasis-indicating [5] laboratory parameters, most commonly $\gamma$-glutamyl transferase and alkaline phosphatase, can be found. In jaundiced patients bilirubin is elevated, with the conjugated form exceeding unconjugated bilirubin [2].

\section{Biomarkers}

Serum carbohydrate antigen 19-9 and carcinoembryonic antigen are commonly used as screening instruments in cholestatic patients, but they are not specific for malignant causes of cholestasis.

Many other serum proteins have been tested as biomarkers but are not used in clinical practice for early detection or diagnosis of CCA.

More recent studies have suggested a number of potential candidates such as microRNA, extracellular vesicles, and circulating tumor DNA but, to date, none has gained sufficient importance for recommendation in clinical practice [5].

There is some evidence from case-control studies for specific peptide marker signatures in both bile and urine of primary sclerosing cholangitis patients suffering from CCA; however, the pathophysiological pathways are yet to be fully elucidated $[6,7]$.

\section{Imaging}

There is no single imaging modality with sufficient accuracy for diagnosis and staging of malignant hilar tumors [8].

Bedside transabdominal ultrasound is usually the first imaging applied to patients with suspicion of biliary disease. It has a high accuracy for detection of dilated bile ducts, the level of obstruction within the biliary tree and mass-forming tumors, and it can help to identify lobar atrophy, aiding the diagnosis of obstructive jaundice [9]. Evaluation of hepatic strictures, however, is difficult in the most frequent case of non-mass-forming pCCA. Most notably in patients with primary sclerosing cholangitis, transabdominal ultrasound is not sufficient to distinguish between inflammatory and malignant causes of biliary obstruction [10].
Contrast-enhanced CT is the most frequently applied imaging method for the assessment of hilar strictures as it evaluates the primary tumor, its adjacent vascular structures (i.e., the hepatic artery and portal vein) and lymph nodes in the abdomen [11]. The best results are obtained with multiphasic CT, including a precontrast scan, a postcontrast (arterial and portal venous) scan, and a late-phase scan. Detection of pCCA is possible with 75-79\% sensitivity and $79-80 \%$ specificity using multiphasic CT $[10,12]$.

Portal vein involvement of a perihilar tumor can thus be diagnosed with a sensitivity of $89 \%$ and a specificity of $92 \%$, whereas hepatic artery involvement can be determined with $84 \%$ sensitivity and $93 \%$ specificity [11]. The accuracy of resectability prediction is not as good, mainly because of frequent underestimation of the lateral tumor spread along the bile ducts [13].

Magnetic resonance tomography (MRI), including magnetic resonance cholangiography, is commonly used in patients with suspected biliary disease and it is the noninvasive standard in differential diagnosis of bile duct strictures because of its sensitivity and specificity to detect pCCA (i.e., $88-89$ and $75-85 \%$, respectively) $[10,12]$. One advantage is the possibility to delineate the complete biliary anatomy in magnetic resonance cholangiography in combination with detection of intra- and hepatic metastases in contrast-enhanced MRI.

Fluorine 18 fluorodeoxyglucose (18F-FDG) PET/CT is of limited use for the assessment of suspected hilar malignancy as the distinction between inflammatory and neoplastic causes often cannot be made with sufficient accuracy and the detection of distant metastases does not exceed that of a multiphasic CT scan [14].

The impact of whole-body $18 \mathrm{~F}-\mathrm{FDG}$ PET/MRI still is under evaluation, and the modality has no established role in the diagnosis and staging of pCCA to date [15].

\section{Direct Cholangiographic Imaging and Tissue Acquisition}

Noninvasive differential diagnosis of hilar strictures is difficult, as described above. Direct cholangiography may add information, but it does not offer sufficient differentiation between benign and malignant diseases.

Biliary strictures can be approached directly via endoscopic retrograde cholangiography (ERC) or percutaneous transhepatic cholangiography (Fig. 1, 2). Both methods allow tissue sampling either by cytology brushing or forceps biopsy of strictured ductal segments as well as drainage of congested bile ducts by stent implantation or percutaneous drainage. The disadvantage of direct cholangiography in comparison to noninvasive imaging is a substantial risk of complications including acute pancreatitis, cholangitis, bleeding, and perforations. 
Fig. 1. ERC: Hilar stricture extending to both liver lobes (pCCA type Klatskin 4).

Fig. 2. Percutaneous transhepatic cholangiography and drainage (PTCD) (right liver lobe) in a patient with a malignant hilar obstruction.
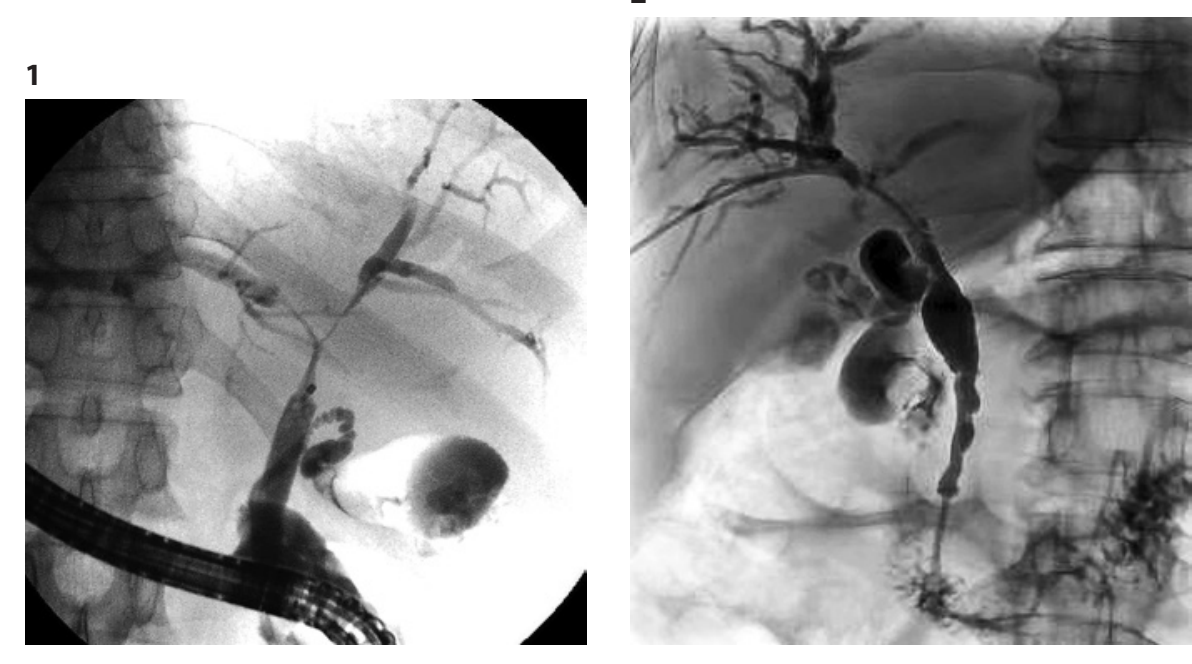

In the majority of cases, tissue acquisition prior to treatment is necessary for the following reasons:

- Even after a thorough preoperative imaging work-up of hilar strictures, in liver resections performed for presumed CCA there is a substantial fraction of patients (i.e., up to $22 \%$ ) who are found to have suffered from benign disease on postoperative evaluation of the resected specimen [16].

- Most patients with pCCA are not surgical candidates either because of an advanced tumor stage or because of physical unfitness for large liver resections. Systemic treatment, on the other hand, will often not be considered feasible before histological and genetic evaluation of the tumor in order to guide conventional and/ or targeted treatment.

Histologic diagnosis of pCCA from endoscopically harvested samples can be rather difficult because of the amount of desmoplastic stroma with relatively few viable cells in many of these tumors. Brushing of biliary strictures to obtain cytological samples is widely practiced, but the sensitivity is rather low (i.e., $35-50 \%)$ [17, 18]. Larger biopsies can usually be obtained by using standard endoscopic forceps to extract transpapillary biopsies of the hepatic hilum under fluoroscopic guidance, with a sensitivity for malignancy of approximately $40-60 \%$ [19, $20]$. Better results are possible when cyto-brushing and acquisition of trans-papillary biopsies are combined [21].

Since the majority of CCA show cellular aneuploidy (an abnormal number of chromosomes within a cell: trisomy, tetrasomy, or polysomy), fluorescence in situ hybridization (FISH) can be used to test single cells from biliary brushings or biopsies for chromosomal aberrations. In patients with de novo hilar strictures without preexisting biliary disease, polysomy is diagnostic for CCA. In patients with inflammatory biliary disease (such as primary sclerosing cholangitis), aneuploidy may indicate biliary dysplasia preceding CCA [22].
Another promising method to enhance tissue-based diagnosis of biliary malignancy is parallel genomic sequencing (next-generation sequencing), a technology that facilitates rapid detection of genomic alterations in biliary epithelial cells. In addition to increasing the sensitivity of the pathological evaluation, it may also provide information guiding toward effective targeted treatment options [23].

To increase the diagnostic yield of both endoscopic imaging and tissue sampling, cholangioscopy with cholangioscopy-guided biopsies is standard diagnostic procedures in suspected hilar malignancy (Fig. 3, 4).

Direct peroral cholangioscopy (DPOCS) with ultraslim gastroscopes or dedicated cholangioscopes offers the advantage of a relatively high resolution due to the large imaging chip, but it has a limited depth of insertion into the bile duct and passage through hilar strictures is frequently not possible [24]. Biopsies through the comparatively large working channels $(2 \mathrm{~mm})$ of these endoscopes can be obtained using standard endoscopic forcepses.

A commonly used alternative to DPOCS are single-use digital cholangioscopes inserted through the working channel of a duodenoscope. These scopes with a smaller outer diameter of $3 \mathrm{~mm}$ and 4-way tip deflection can often be passed through (malignant) hilar strictures and thus can help estimate the intrahepatic tumor extent. Tissue sampling is limited to dedicated small-caliber $(1 \mathrm{~mm})$ forceps and it is frequently more cumbersome than in DPOCS. By using cholangioscopy to guide tissue acquisition of hilar strictures, the sensitivity for malignancy can be increased up to $76.5 \%$ with a negative predictive value of $69.2 \%$ [25].

Although tissue sampling from hilar strictures still is considered the standard of diagnosis, cholangioscopic evaluation and classification using high-quality imaging is gaining importance. Inclusion of visual inspection of biliary alterations in the diagnostic algorithm can result 

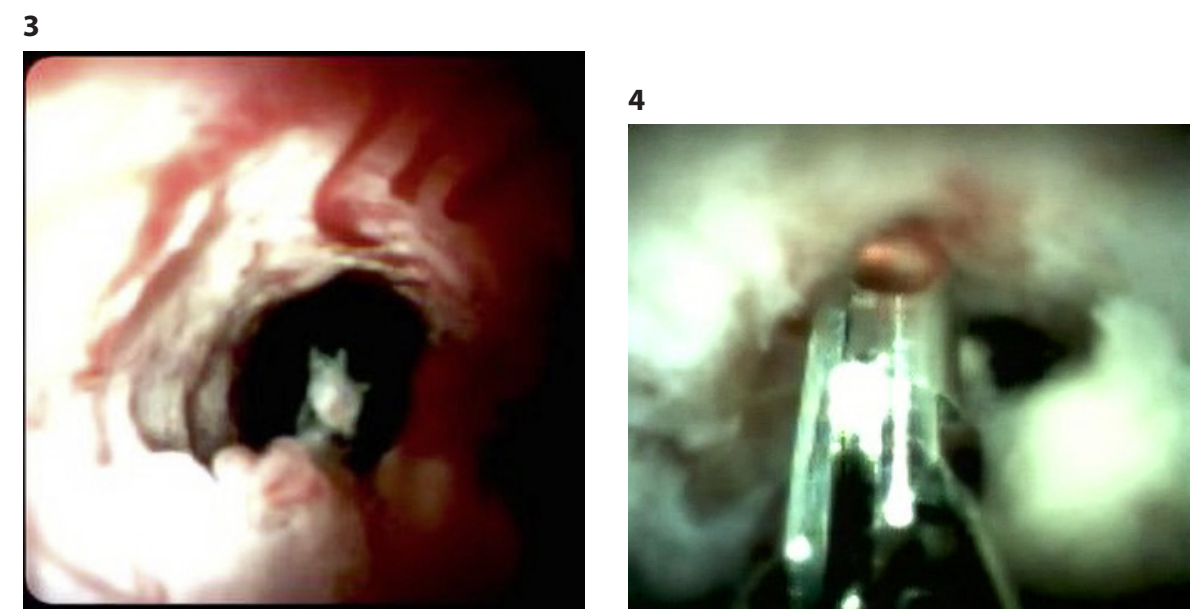

Fig. 3. Cholangioscopic appearance of a malignant hilar stricture with visible tumor vessels.

Fig. 4. Cholangioscopically guided biopsy of a suspicious intraductal lesion.

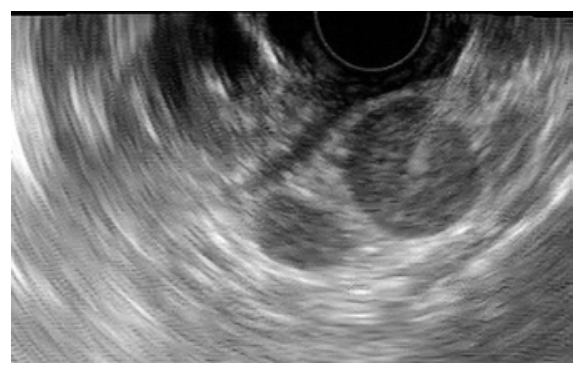

Fig. 5. Periportal lymph nodes (during EUS-guided FNA).

in a higher accuracy of malignancy diagnosis than tissue evaluation alone (93 vs. 89\%) [26]. Problematic in this regard is the lack of broadly accepted criteria for diagnosing malignancy in the cholangioscopic (i.e., visual) evaluation of biliary strictures. Neither vascular nor epithelial alterations of bile duct surfaces offer a high enough specificity for diagnosis or a sufficient negative predictive value for exclusion of biliary cancer [27, 28]. Accurate staging of the local tumor extent in intrahepatic ducts by cholangioscopy - intended to guide the decision on resectability before laparotomy - is challenging because of the commonly present cancer spread beneath the surface of bile ducts along periductal structures [29].

\section{Endoscopic Ultrasound}

Endoscopic ultrasound (EUS) is currently used for both detection of perihilar tumor masses and locoregional staging of lymphonodular spread [30].

Sensitivity for detection of perihilar cancer is $>80 \%$, but it is lower than for distal CCA [31]. However, while the extrahepatic ductal structures can easily be evaluated, complete tumor staging is often precluded by the limited visualization of the hilar region; portal vein invasion, for instance, is only detectable with EUS in $57 \%$ of affected cases [32].

Tissue acquisition via EUS through fine-needle aspiration (FNA) or fine-needle biopsy is technically possible in a majority of patients with suspected perihilar malignancy. In a metanalysis pooling 6 studies the sensitivity was $66 \%$, with a $95 \%$ CI of $57-74 \%$ [33]. This endosonographically guided approach to diagnosis, however, bears a substantial risk. In a series of patients undergoing EUS-guided FNA of hilar CCA, 5 out of 6 patients $(83 \%)$ with a positive FNA later showed peritoneal metastases on diagnostic laparoscopy performed for a liver transplant evaluation (compared to only $8 \%$ without a prior FNA), indicating a high risk of tumor dissemination [34]. In patients not undergoing liver transplantation - clearly the majority of cases - however, FNA does not seem to negatively affect treatment outcomes [35].

Additionally, EUS seems to have a high sensitivity for locoregional lymph node metastases of pCCA, with an acceptable negative predictive value [30] (Fig. 5).

\section{Intraductal Ultrasound}

Intraductal ultrasound (IDUS) is performed by inserting a thin ultrasound probe with a scanning frequency of $15-20 \mathrm{MHz}$ over a guide wire into the biliary tract, typically during an endoscopic retrograde cholangiopancreatography procedure. Alterations of the ductal wall such as focal thickening, and concentric and excentric strictures, as well as deep ductal ulcerations can be visualized, while nodal involvement can infrequently be diagnosed owing to the very limited depth of ultrasound imaging (Fig. 6).

A German research group reported a sensitivity of $98 \%$ in diagnosing CCA $(n=85)$, with a specificity of $98 \%$ and an accuracy of $92 \%$. Of note, the investigators were not blinded to clinical and other imaging data [36]. 


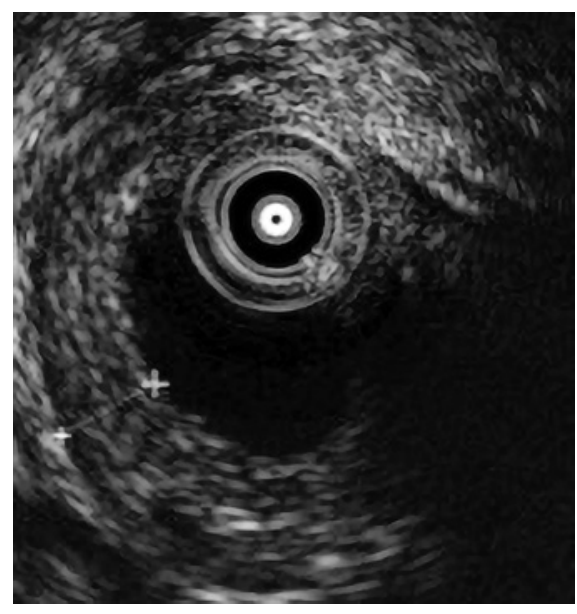

Fig. 6. IDUS of a smooth excentric biliary stricture without distinct features of malignancy.

The diagnostic accuracy of IDUS for CCA diagnosis is decreased in bile duct segments pretreated with (balloon) dilation or stents.

\section{Confocal Laser Endomicroscopy}

Confocal laser endomicroscopy (CLE) is a probe-based endoscopic technique for visualization of epithelia in vivo. After identification of suspicious biliary strictures on conventional imaging (i.e., ERC), direct contact of the probe (inserted through the working channel of a duodenoscope) with the lesion is made. The technology uses a low-power laser to illuminate tissue surfaces after injection of fluorescein with collection of reflected fluorescent light spectra to create high-resolution images [37]. It is possible to distinguish single epithelial cells using CLE in the biliary tract and thus efforts have been made to devise classifications aimed at standardization of CLE findings [38, 39]. In indeterminate biliary strictures, CLE showed a higher sensitivity (89\%) and accuracy (82\%) for diagnosis of malignancy than standard cyto- and histopathology (56 and $72 \%$, respectively) [40]. Still, the technical requirements for CLE are complex and there remains a remarkably high interobserver variability on the interpretation of CLE findings despite attempts at standardization, thus leaving questions regarding future dissemination of the technique.

\section{Staging Laparoscopy}

(Routine) staging laparoscopy prior to surgical treatment of pCCA aims at excluding liver metastases and peritoneal spread undetected by cross-sectional imaging (and PET).

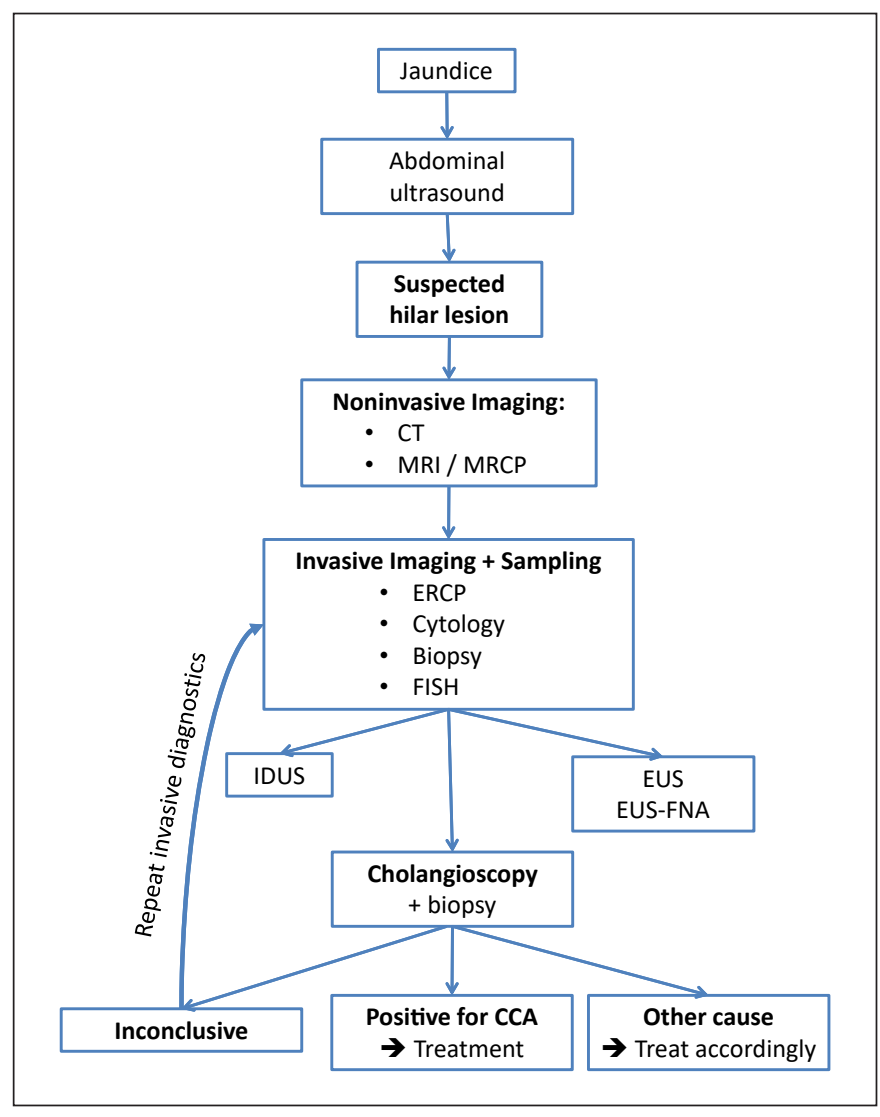

Fig. 7. Diagnostic workup in patients with a suspected hilar malignancy.

Although it is a long-standing tradition in a number of centers, its role has decreased in recent years because of a relatively low diagnostic yield even with the addition of laparoscopic ultrasound and the increasing importance of the other diagnostic procedures described above [41].

An algorithm for the diagnostic workup of a suspected hilar malignancy is given in Figure 7.

\section{Conclusion}

Jaundice resulting from perihilar bile duct strictures is often the clinical presentation of pCCA, mostly arising in patients without known risk factors for this tumor.

Diagnosis of pCCA is often difficult because of the lack of distinctive features in laboratory parameters and on cross-sectional and ultrasound imaging. A mosaic of diagnostic tests can strengthen the presumptive diagnosis.

Direct imaging has improved in recent years with the emergence of relatively new technologies such as digital cholangioscopy and endomicroscopy, but the mainstay of accurate diagnosis of perihilar malignancy remains tissue sampling. 
Cytological and histological specimens harvested via ERC in combination with cholangioscopy are the most commonly used for establishing a definite diagnosis of malignant biliary strictures, but the low sensitivity in the presence of paucicellular and desmoplastic tumors accounts for time-consuming repetitions of diagnostic steps.

\section{Conflict of Interest Statement}

The authors declare that there are no conflict of interests.

\section{Funding Sources}

The authors declare that they received no funding related to this review.

\section{Author Contributions}

E.S. drafted this work, contributed substantially to the literature review, revised this work, and approved the final version. A.D. revised this work, completed the literature review, contributed the figures, and approved the final version.

\section{References}

1 Aljiffry M, Abdulelah A, Walsh M, Peltekian K, Alwayn I, Molinari M. Evidence-based approach to cholangiocarcinoma: a systematic review of the current literature. J Am Coll Surg. 2009 Jan;208(1):134-47.

2 Khan SA, Davidson BR, Goldin RD, Heaton N, Karani J, Pereira SP, et al.; British Society of Gastroenterology. Guidelines for the diagnosis and treatment of cholangiocarcinoma: an update. Gut. 2012 Dec;61(12):1657-69.

3 Yachimski P, Pratt DS. Cholangiocarcinoma: natural history, treatment, and strategies for surveillance in high-risk patients. J Clin Gastroenterol. 2008 Feb;42(2):178-90.

4 Blechacz B, Komuta M, Roskams T, Gores GJ. Clinical diagnosis and staging of cholangiocarcinoma. Nat Rev Gastroenterol Hepatol. 2011 Aug;8(9):512-22.

5 Nault J-C, Villanueva A. Biomarkers for hepatobiliary cancers. Hepatology. 2020. doi: 10.1002/hep.31175.

6 Lankisch TO, Metzger J, Negm AA, Vosskuhl $\mathrm{K}$, Schiffer E, Siwy J, et al. Bile proteomic profiles differentiate cholangiocarcinoma from primary sclerosing cholangitis and choledocholithiasis. Hepatology. 2011 Mar;53(3): 875-84.

7 Metzger J, Negm AA, Plentz RR, Weismüller TJ, Wedemeyer J, Karlsen TH, et al. Urine proteomic analysis differentiates cholangiocarcinoma from primary sclerosing cholangitis and other benign biliary disorders. Gut. 2013 Jan;62(1):122-30.

8 Hennedige TP, Neo WT, Venkatesh SK. Imaging of malignancies of the biliary tract: an update. Cancer Imaging. 2014 Apr; 14:14.

9 Olthof SC, Othman A, Clasen S, Schraml C, Nikolaou K, Bongers M. Imaging of Cholangiocarcinoma. Visc Med. 2016 Dec;32(6): 402-10.

10 Charatcharoenwitthaya P, Enders FB, Halling $\mathrm{KC}$, Lindor KD. Utility of serum tumor markers, imaging, and biliary cytology for detecting cholangiocarcinoma in primary sclerosing cholangitis. Hepatology. 2008 Oct;48(4): 1106-17.

11 Ruys AT, van Beem BE, Engelbrecht MR, Bipat S, Stoker J, Van Gulik TM. Radiological staging in patients with hilar cholangiocarcinoma: a systematic review and meta-analysis. Br J Radiol. 2012 Sep;85(1017):1255-62.
12 Saluja SS, Sharma R, Pal S, Sahni P, Chattopadhyay TK. Differentiation between benign and malignant hilar obstructions using laboratory and radiological investigations: a prospective study. HPB (Oxford). 2007;9(5):37382.

13 Vilgrain V. Staging cholangiocarcinoma by imaging studies. HPB (Oxford). 2008;10(2): 106-9.

14 Annunziata S, Caldarella C, Pizzuto DA, Galiandro F, Sadeghi R, Giovanella L, et al. Diagnostic accuracy of fluorine-18-fluorodeoxyglucose positron emission tomography in the evaluation of the primary tumor in patients with cholangiocarcinoma: a meta-analysis. BioMed Res Int. 2014;2014:247693.

15 Kirchner J, Sawicki LM, Deuschl C, Grüneisen J, Beiderwellen K, Lauenstein TC, et al. 18 F-FDG PET/MR imaging in patients with suspected liver lesions: value of liver-specific contrast agent Gadobenate dimeglumine. PLoS One. 2017 Jul;12(7):e0180349.

16 Hubers LM, Maillette de Buy Wenniger LJ, Doorenspleet ME, Klarenbeek PL, Verheij J, Rauws EA, et al. IgG4-associated cholangitis: a comprehensive review. Clin Rev Allergy Immunol. 2015 Jun;48(2-3):198-206.

17 Adamsen S, Olsen M, Jendresen MB, Holck S, Glenthøj A. Endobiliary brush biopsy: Intraand interobserver variation in cytological evaluation of brushings from bile duct strictures. Scand J Gastroenterol. 2006 May;41(5): 597-603.

18 Ponchon T, Gagnon P, Berger F, Labadie M, Liaras A, Chavaillon A, et al. Value of endobiliary brush cytology and biopsies for the diagnosis of malignant bile duct stenosis: results of a prospective study. Gastrointest Endosc. 1995 Dec;42(6):565-72.

19 Weber A, von Weyhern C, Fend F, Schneider J, Neu B, Meining A, et al. Endoscopic transpapillary brush cytology and forceps biopsy in patients with hilar cholangiocarcinoma. World J Gastroenterol. 2008 Feb;14(7):1097101.

20 Pugliese V, Conio M, Nicolò G, Saccomanno S, Gatteschi B. Endoscopic retrograde forceps biopsy and brush cytology of biliary strictures: a prospective study. Gastrointest Endosc. 1995 Dec;42(6):520-6.
21 Peter S, Council L, Bang JY, Neumann H, Mönkemüller K, Varadarajulu S, et al. Poor agreement between endoscopists and gastrointestinal pathologists for the interpretation of probe-based confocal laser endomicroscopy findings. World J Gastroenterol. $2014 \mathrm{Dec}$; 20(47):17993-8000.

22 Eaton JE, Gossard AA, Talwalkar JA. Recall processes for biliary cytology in primary sclerosing cholangitis. Curr Opin Gastroenterol. 2014 May;30(3):287-94.

23 Singhi AD, Nikiforova MN, Chennat J, Papachristou GI, Khalid A, Rabinovitz M, et al. Integrating next-generation sequencing to endoscopic retrograde cholangiopancreatography (ERCP)-obtained biliary specimens improves the detection and management of patients with malignant bile duct strictures. Gut. 2020 Jan;69(1):52-61.

24 Parsa N, Khashab MA. The Role of Peroral Cholangioscopy in Evaluating Indeterminate Biliary Strictures. Clin Endosc. 2019 Nov; 52(6):556-64

25 Draganov PV, Chauhan S, Wagh MS, Gupte AR, Lin T, Hou W, et al. Diagnostic accuracy of conventional and cholangioscopy-guided sampling of indeterminate biliary lesions at the time of ERCP: a prospective, long-term follow-up study. Gastrointest Endosc. 2012 Feb;75(2):347-53.

26 Ogura T, Imanishi M, Kurisu Y, Onda S, Sano T, Takagi W, et al. Prospective evaluation of digital single-operator cholangioscope for diagnostic and therapeutic procedures (with videos). Dig Endosc. 2017 Nov;29(7):782-9.

27 Seo DW, Lee SK, Yoo KS, Kang GH, Kim MH, Suh DJ, et al. Cholangioscopic findings in bile duct tumors. Gastrointest Endosc. 2000 Nov; 52(5):630-4.

28 Fukuda Y, Tsuyuguchi T, Sakai Y, Tsuchiya S, Saisyo H. Diagnostic utility of peroral cholangioscopy for various bile-duct lesions. Gastrointest Endosc. 2005 Sep;62(3):374-82.

29 Lewis HL, Rahnemai-Azar AA, Dillhoff M, Schmidt CR, Pawlik TM. Current Management of Perihilar Cholangiocarcinoma and Future Perspectives. Chirurgia (Bucur). 2017 May-Jun;112(3):193-207.

30 Gleeson FC, Rajan E, Levy MJ, Clain JE, Topazian MD, Harewood GC, et al. EUS-guided FNA of regional lymph nodes in patients with unresectable hilar cholangiocarcinoma. Gastrointest Endosc. 2008 Mar;67(3):438-43. 
31 Mohamadnejad M, DeWitt JM, Sherman S, LeBlanc JK, Pitt HA, House MG, et al. Role of EUS for preoperative evaluation of cholangiocarcinoma: a large single-center experience. Gastrointest Endosc. 2011 Jan;73(1):71-8.

32 Tamada $\mathrm{K}$, Ido $\mathrm{K}$, Ueno $\mathrm{N}$, Ichiyama $\mathrm{M}$, Tomiyama T, Nishizono T, et al. Assessment of portal vein invasion by bile duct cancer using intraductal ultrasonography. Endoscopy. 1995 Oct;27(8):573-8.

33 Navaneethan U, Njei B, Venkatesh PG, Lourdusamy V, Sanaka MR. Endoscopic ultrasound in the diagnosis of cholangiocarcinoma as the etiology of biliary strictures: a systematic review and meta-analysis. Gastroenterol Rep (Oxf). 2015 Aug;3(3):209-15.

34 Heimbach JK, Sanchez W, Rosen CB, Gores GJ. Trans-peritoneal fine needle aspiration biopsy of hilar cholangiocarcinoma is associated with disease dissemination. HPB (Oxford). 2011 May;13(5):356-60.
35 El Chafic AH, Dewitt J, Leblanc JK, El Hajj II, Cote G, House MG, et al. Impact of preoperative endoscopic ultrasound-guided fine needle aspiration on postoperative recurrence and survival in cholangiocarcinoma patients. Endoscopy. 2013 Nov;45(11):883-9.

36 Meister T, Heinzow HS, Woestmeyer C, Lenz P, Menzel J, Kucharzik T, et al. Intraductal ultrasound substantiates diagnostics of bile duct strictures of uncertain etiology. World J Gastroenterol. 2013 Feb;19(6):874-81.

37 Kantsevoy SV, Adler DG, Conway JD, Diehl DL, Farraye FA, Kaul V, et al.; ASGE Technology Committee. Confocal laser endomicroscopy. Gastrointest Endosc. 2009 Aug;70(2): $197-200$
38 Meining A, Shah RJ, Slivka A, Pleskow D, Chuttani R, Stevens PD, et al. Classification of probe-based confocal laser endomicroscopy findings in pancreaticobiliary strictures. Endoscopy. 2012 Mar;44(3):251-7.

39 Caillol F, Filoche B, Gaidhane M, Kahaleh M. Refined probe-based confocal laser endomicroscopy classification for biliary strictures: the Paris Classification. Dig Dis Sci. 2013 Jun 58(6):1784-9.

40 Slivka A, Gan I, Jamidar P, Costamagna G, Cesaro P, Giovannini M, et al. Validation of the diagnostic accuracy of probe-based confocal laser endomicroscopy for the characterization of indeterminate biliary strictures: results of a prospective multicenter international study. Gastrointest Endosc. 2015 Feb;81(2): 282-90.

41 Ruys AT, Busch OR, Gouma DJ, van Gulik TM. Staging laparoscopy for hilar cholangiocarcinoma: is it still worthwhile? Ann Surg Oncol. 2011 Sep;18(9):2647-53. 